\title{
Quantitative Modeling of Kinetically Controlled Nanocrystal Synthesis with Liquid Cell Electron Microscopy
}

\author{
Mei Wang, ${ }^{1}$ Taylor J. Woehl ${ }^{1}$ and Chiwoo Park $^{2}$ \\ 1. Department of Chemical and Biomolecular Engineering, University of Maryland, College Park, \\ MD, USA \\ 2. Department of Industrial and Manufacturing Engineering, Florida State University, Tallahassee, \\ FL, USA
}

It is widely known that the properties of nanomaterials depend on their size and shape [1]. However, due to a lack of experimental characterization techniques for probing their formation dynamics, the nucleation and subsequent growth mechanisms that control the shape and size of nanocrystals are still poorly understood. With the advent of in-situ liquid cell electron microscopy (LCEM), nucleation and growth process can be observed in real time at nanometer scale resolution [2]. In liquid cell experiments, the electron beam can serve as both the stimulus to induce nucleation and the imaging tool. Currently, analysis of nucleation and growth kinetics by LCEM is semi-quantitative, because there exist multiple forward and backward reactions and there is poor kinetic control over the experiments. This there is a critical need to bring quantitative reaction kinetics and quantitative modeling of nucleation and growth to electron beam induced growth with LCEM.

Here we show that silver nanoparticles can be synthesized under strict kinetic control by applying radical scavengers and utilizing a micro-fluidic system during LCEM experiments to enable quantitative modeling of nucleation and growth mechanisms. When the electron beam irradiates water or an aqueous solution, water molecules break down into several radicals and molecular species. Among these species, aqueous electrons and hydrogen radicals are strong reducing species that can reduce metal ions to metal atoms [3]. However, oxidizing species $\left(\mathrm{OH}^{\circ}, \mathrm{O}_{2}\right)$ are produced at the same time, which oxidize metal atoms back to higher valences [4]. To eliminate oxidizing reactions, establish purely reducing species, and enhance reproducibility, we have added tertiary-butanol, a typical $\mathrm{OH}^{\circ}$ and $\mathrm{O}_{2}$ scavenger [5]. Parameters such as magnification, spot size, dwell time were changed to tune the dose rate in the system. Image analysis using Matlab code was employed to track the nanoparticles formed in real time to quantify the nucleation kinetics as a function of dose rate. Scanning electron microscopy (SEM) and high-resolution transmission electron microscopy (HRTEM) were used to characterize the resulting atomic structure, morphology, and size of silver nanoparticles.

An example of time lapse series of bright field STEM images of Ag nanoparticle formation (Figure 1) showed that the numbers of silver particles in the viewing area increased with time, with distinct changes in the slope (red lines, Figure 1). The different slopes of numbers of particle vs time is hypothesized to result from different types of heterogeneous nucleation sites on the SiN membrane with different activation energies. Under this experimental condition, particles with different morphologies were produced by the electron beam. Ex-situ SEM images (Figure 2a) showed that spherical, rod-shaped, and nanoplates were formed on the SiN membrane during the STEM liquid cell experiments. The spherical particles range from around 50-100 $\mathrm{nm}$ in diameter while the rod-shaped particles range from $200-400 \mathrm{~nm}$ in length while the diameters were approximately 50-100 $\mathrm{nm}$. The SEM results correlate with the previous results from the time lapse series of STEM images that also showed similar morphology. HRTEM images (Figure 2b) showed that in addition to the larger 
spherical silver particles seen in SEM images, particles as small as $10 \mathrm{~nm}$ also formed during LCEM experiments. Lattice planes and multiple twin boundaries can clearly be seen in HRTEM images of the silver nanoparticles, indicating they are crystalline [6].

\section{References:}

[1] Xia, Y. et al, Chem. Int. Ed. 48(1) (2009), p. 60.

[2] Zheng, H. et al, Science 324(5932) (2009), p. 1309.

[3] Woehl, T. J. et al, ACS Nano 6(10) (2012), p. 8599.

[4] Schneider, N. M. et al, J. Phys. Chem. C 118(38) (2014), p. 22373.

[5] Woehl, T. J., \& Abellan, P. J. Microscopy, 265(2) (2017), p. 135.

[6] T.J.W. acknowledges funding from Oak Ridge Associated Universities. M.W. acknowledges a fellowship from University of Maryland Energy Research Center.
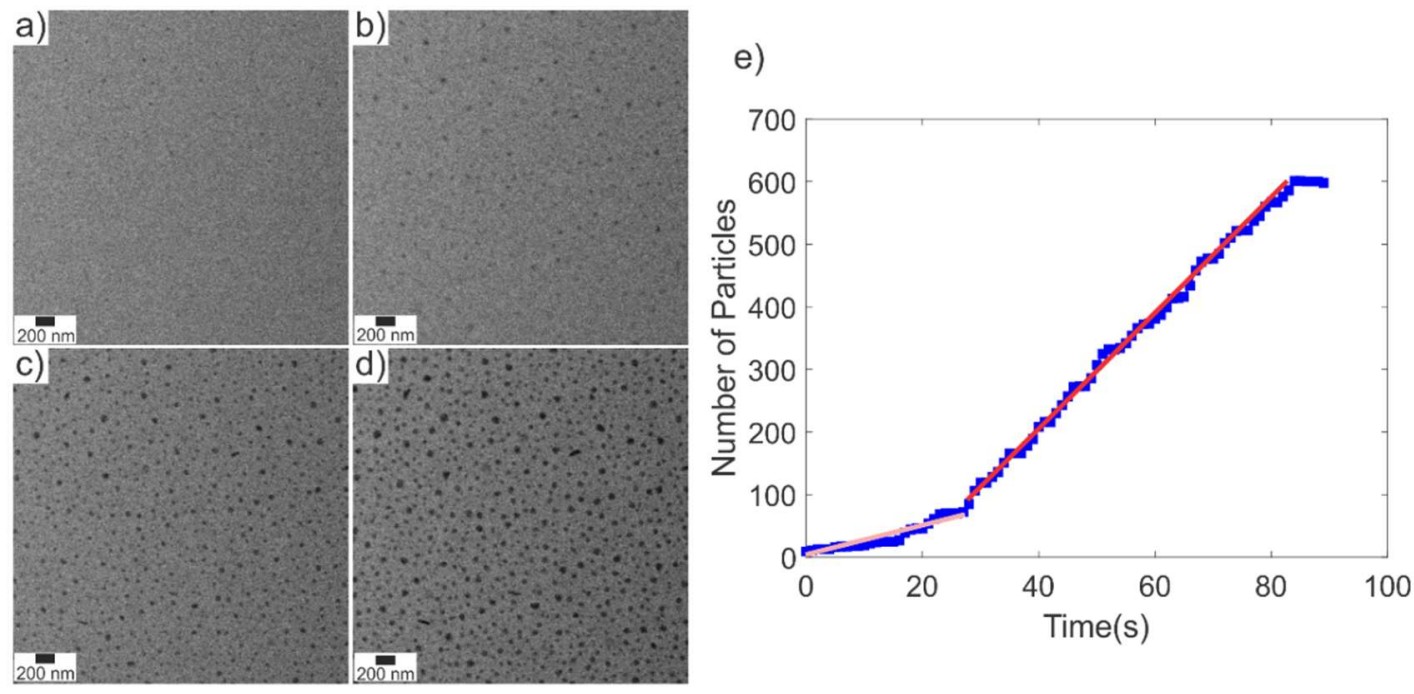

Figure 1. An example of time lapse series of bright field STEM images of Ag, the magnification is $100 \mathrm{k}$, current density is $31 \mathrm{pA}$, image size 1024 x 1024 pixels, yielding dose rate to be $1.329 \cdot 10^{6}$ $\mathrm{Gy} / \mathrm{s}$, dwell time is $5 \mu \mathrm{s}$. a)-d) $0 \mathrm{~s}, 25 \mathrm{~s}, 50 \mathrm{~s}, 85 \mathrm{~s}$ and e) Number of particles as a function of time.
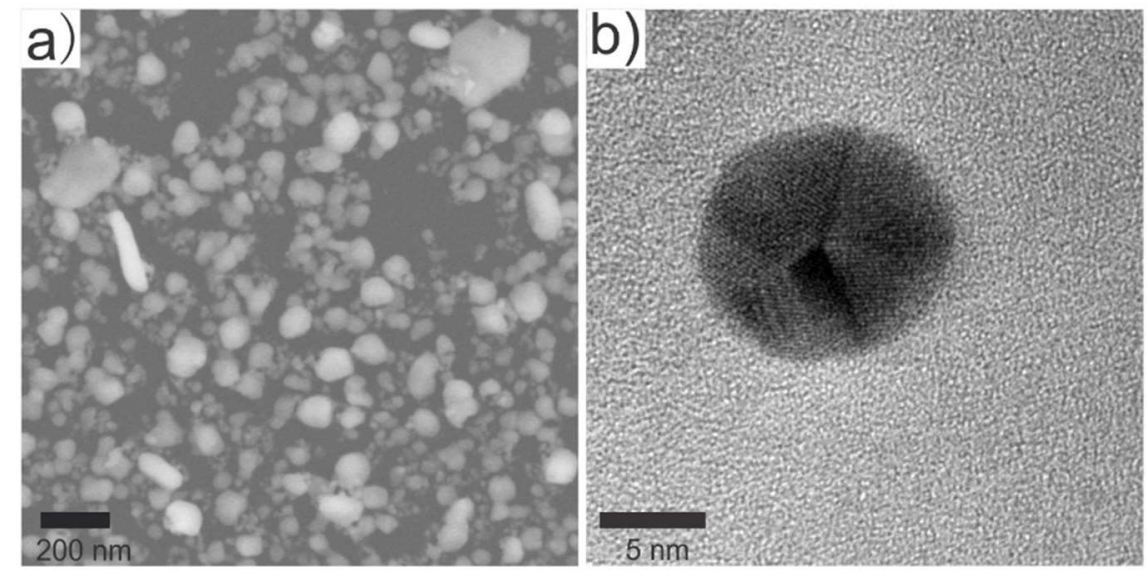

Figure 2. a) Ex situ SEM and b) HRTEM images of Ag nanoparticles on SiN membrane after LCEM experiments. 